\title{
Stochastic Phase Field Equations: Existence and Uniqueness
}

\author{
L. Bertini*, S. Brassesco, P. Buttà̀ and E. Presutti ${ }^{\dagger}$
}

\begin{abstract}
We consider a conservative system of stochastic PDE's, namely a one dimensional phase field model perturbed by an additive space-time white noise. We prove a global existence and uniqueness result in a space of continuous functions on $\mathbb{R}_{+} \times \mathbb{R}$. This result is obtained by extending previous results of Doering [3] on the stochastic Allen-Cahn equation.
\end{abstract}

\section{Introduction and results}

We consider a phase field system with additive stochastic noise, which is formally written as

$$
\begin{aligned}
& \partial_{t} m(t)=\frac{1}{2} \Delta m(t)-V^{\prime}(m(t))+\lambda h(t)+a \eta(t) \\
& \partial_{t}[h(t)+m(t)]=\frac{1}{2} \Delta h(t)
\end{aligned}
$$

where $(t, x) \in \mathbb{R}_{+} \times \mathbb{R}, m(t)=m(t, x), h(t)=h(t, x)$ are two scalar random fields (we omit to write explicitly the dependence on the randomness), $\lambda$ is a positive parameter, $\Delta$ is the Laplacian on $\mathbb{R}, V(m)=m^{4} / 4-m^{2} / 2$ is a double well potential, $a=a(x)$ is a bounded and continuous function, and $\eta(t)=\eta(t, x)$ is a space-time white noise, i.e. $\mathbb{E}(\eta(t, x) \eta(s, y))=\delta(t-s) \delta(x-y)$. In particular a translationally covariant noise is obtained for $a=1$.

The deterministic system obtained by setting $a=0$ in (1.1), usually referred to as phase field equations, describes the kinetic of phase segregation when the presence of the latent heat is taken into account. The first equation describes in fact the evolution of the order parameter $m$ which is coupled to the external field $h$ (which can be thought as the excess temperature measured from the melting temperature); $h$ is however itself a dynamic variable which diffuses and, via the coupling $\lambda$, introduces a feedback into $m$ whose effect is to slow down the phase segregation process. We stress that $q=m+h$ is locally conserved as apparent from the second equation in (1.1). Scaling limits of the deterministic phase field equations as $\lambda \rightarrow 0$ have been considered in [2] and [6].

\footnotetext{
*Partially supported by Cofinanziamento MURST 1999.

†Partially supported by Cofinanziamento MURST 1999 and by NATO Grant PST.CLG. 976552.
} 
Due to the phenomenological character of the phase field model, the introduction of a random forcing term appears natural and makes possible to discuss statistical properties of solutions. We also mention that the stochastic system (1.1) is very similar to the so-called, in the physical literature on critical phenomena, model $\mathrm{C}$ of Hohenberg and Halperin [5]. The specific choice of an additive white noise only in the first equation has been done to keep the model as simple as possible: the random forcing term is non-conservative, whereas the conservation law is still linear and not perturbed. Moreover, for $\lambda$ small, $m$ and $h$ are weakly coupled so that we may refer to (1.1) as a weakly conservative system. This simplifying feature might help in developing a mathematical theory for phase segregation in conservative models. Indeed, in the companion paper [1], front fluctuations for (1.1) are analyzed in a suitable scaling limit as $\lambda \rightarrow 0$ and $a=O(\lambda)$. The need of an existence and uniqueness result for the stochastic system (1.1) in [1] is the main motivation for the present paper.

Referring to [1] for a more exhaustive discussion on the stochastic phase field equations, we next state precisely our results. For $\alpha>0$ and $\gamma \in(0,1]$, let us define the following norms on $C(\mathbb{R})$ :

$$
\begin{aligned}
\|\varphi\|_{C_{\alpha}(\mathbb{R})} & :=\sup _{x \in \mathbb{R}} e^{-\alpha|x|}|\varphi(x)| \\
\|\varphi\|_{C_{\alpha}^{\gamma}(\mathbb{R})} & :=\|\varphi\|_{C_{\alpha}(\mathbb{R})}+\sup _{x \neq y} e^{-\alpha(|x|+|y|)} \frac{|\varphi(x)-\varphi(y)|}{|x-y|^{\gamma}}
\end{aligned}
$$

and also the following ones on $C\left(\mathbb{R}_{+} \times \mathbb{R}\right)$

$$
\begin{aligned}
\|f\|_{C_{\alpha}\left(\mathbb{R}_{+} \times \mathbb{R}\right)} & :=\sup _{t \in \mathbb{R}_{+}} e^{-\alpha^{2} t / 2}\|f(t)\|_{C_{\alpha}(\mathbb{R})} \\
\|f\|_{C_{\alpha}^{\gamma}\left(\mathbb{R}_{+} \times \mathbb{R}\right)} & :=\sup _{t \in \mathbb{R}_{+}} e^{-\alpha^{2} t / 2}\|f(t)\|_{C_{\alpha}^{\gamma}(\mathbb{R})}
\end{aligned}
$$

we shall denote by $C_{\alpha}(\mathbb{R}), \ldots$, the corresponding Banach spaces.

Let $p_{t}=e^{t \Delta / 2}$ be the heat semigroup, namely the integral operator with kernel $p_{t}(x, y)=(2 \pi t)^{-1 / 2} \exp \left\{-(x-y)^{2} / 2 t\right\}$. We introduce the process $Z(t)=$ $Z(t, x)$ given by $Z(t)=\int_{0}^{t} d s p_{t-s}[a \eta(s)]$. Then $Z$ is the mean zero Gaussian process with covariance

$$
\mathbb{E}(Z(t, x) Z(s, y))=\Gamma(t, s ; x, y):=\int_{0}^{t \wedge s} d u \int d z p_{t-u}(x-z) p_{s-u}(y-z) a(z)^{2}
$$

where $t \wedge s:=\min \{t, s\}$. In the next lemma we state some properties of the paths of $Z$, which follow, by standard Gaussian arguments, from the properties of the covariance $\Gamma$, see e.g. [3] and references therein.

Lemma 1.1 For each $\alpha>0$ and $\gamma \in(0,1 / 2)$ we have that $Z \in C_{\alpha}^{\gamma}\left(\mathbb{R}_{+} \times \mathbb{R}\right), \mathbb{P}-$ a.s.

We shall denote by $\mathcal{F}_{t}$ the filtration given by $\mathcal{F}_{t}:=\sigma\{Z(s, x) ;(s, x) \in[0, t] \times$ $\mathbb{R}\}$. In the following we consider a fixed realization of $Z \in C_{\alpha}^{\gamma}\left(\mathbb{R}_{+} \times \mathbb{R}\right)$. 
Let $q:=m+h$ and $U^{\prime}(m):=V^{\prime}(m)-\lambda m$; we formulate the problem (1.1) in the integral form

$$
\begin{aligned}
& m(t)=p_{t} m(0)+\int_{0}^{t} d s p_{t-s}\left[-U^{\prime}(m(s))+\lambda q(s)\right]+Z(t) \\
& q(t)=p_{t} q(0)-\frac{1}{2} \int_{0}^{t} d s \Delta p_{t-s} m(s)
\end{aligned}
$$

note that the integral on the r.h.s. of the second equation is well defined (the result is a continuous function) provided $x \mapsto m(s, x)$ is Hölder continuous.

Our main result is the following existence and uniqueness result for the system (1.3) on the space of Hölder continuous functions.

Theorem 1.2 Let $m(0), q(0) \in C_{\alpha}^{\gamma}(\mathbb{R})$ for any $\alpha>0$ and $\gamma \in(0,1 / 2)$. Then there exists a unique $\mathcal{F}_{t}$-adapted process $(m, q) \in C_{\alpha}^{\gamma}\left(\mathbb{R}_{+} \times \mathbb{R}\right) \times C_{\alpha}^{\gamma}\left(\mathbb{R}_{+} \times \mathbb{R}\right)$ for any $\alpha>0$ and $\gamma \in(0,1 / 2)$ which solves (1.3).

We introduce the linear operators $G, \tilde{G}$ on $C_{\alpha}\left(\mathbb{R}_{+} \times \mathbb{R}\right)$ given by

$$
\begin{aligned}
G[F](t) & :=\int_{0}^{t} d s p_{t-s} F(s) \\
\tilde{G}[F](t) & :=\int_{0}^{t} d s(t-s) \frac{1}{2} \Delta p_{t-s} F(s)
\end{aligned}
$$

and set $R(t):=p_{t} m(0)+\lambda t p_{t} q(0)+Z(t)$. By plugging the second equation in (1.3) into the first one we get that $m$ solves the problem

$$
m=R-G\left[U^{\prime}(m)\right]-\lambda \tilde{G}[m] .
$$

By standard estimates on the heat kernel, it is easy to verify that for each $\alpha>0$ and $\gamma \in(0,1]$ there exist an $\alpha^{\prime}>0$ and a constant $C=C(\alpha, \gamma)$ such that

$$
\begin{aligned}
\|G f\|_{C_{\alpha}^{\gamma}\left(\mathbb{R}_{+} \times \mathbb{R}\right)} \leq C\|f\|_{C_{\alpha^{\prime}}\left(\mathbb{R}_{+} \times \mathbb{R}\right)}, \\
\|\tilde{G} f\|_{C_{\alpha}^{\gamma}\left(\mathbb{R}_{+} \times \mathbb{R}\right)} \leq C\|f\|_{C_{\alpha^{\prime}}\left(\mathbb{R}_{+} \times \mathbb{R}\right)} .
\end{aligned}
$$

Furthermore, for each $\alpha>0$ and $\gamma \in(0,1)$, there exists an $\alpha^{\prime}>0, \gamma^{\prime} \in(0,1)$ and a constant $C=C(\alpha, \gamma)$ such that

$$
\left\|\int_{0}^{t} d s \Delta p_{t-s} f(s)\right\|_{C_{\alpha}^{\gamma}\left(\mathbb{R}_{+} \times \mathbb{R}\right)} \leq C\|f\|_{C_{\alpha^{\prime}}^{\gamma^{\prime}\left(\mathbb{R}_{+} \times \mathbb{R}\right)}} .
$$

Therefore Theorem 1.2 can be easily deduced from the following existence and uniqueness result for the problem (1.5) on the space of continuous functions.

Theorem 1.3 Let $m(0), q(0) \in C_{\alpha}(\mathbb{R})$ for any $\alpha>0$. Then there exists a unique $\mathcal{F}_{t}$-adapted process $m \in C_{\alpha}\left(\mathbb{R}_{+} \times \mathbb{R}\right)$ for any $\alpha>0$ which solves (1.5). 
In the rest of the paper we prove Theorem 1.3. A uniqueness and existence result for equation 1.5 with $\lambda=0$ is given in [3]. See also [4] for the analogous result in a bounded domain. We shall follow closely the proof in [3] for the one dimensional case, referring to that paper for some technical Lemmata. The term $\lambda \tilde{G}$, coming from $\lambda h$ in (1.1), is the source of the difficulties. Since we cannot estimate the $L^{p}$ norm of $h$ in terms of the $L^{p}$ norm $m$, the necessary a priori bounds are not a straightforward extension of that in [3]. To overcame this problem we shall estimate an appropriate negative Sobolev norm of $h$ in terms of the $L^{p}$ norm of $m$, see Lemmata 2.4 and 2.5 below.

We finally remark that the quartic double well potential, $V(m)=m^{4} / 4-$ $\mathrm{m}^{2} / 2$ has been chosen only for notation simplicity; the proof works for any polynomial of even degree with positive leading coefficient.

Since the parameter $\lambda$ will be kept fixed throughout all the paper we omit to indicate the dependence on it. We shall denote by $C$ a generic positive constant whose numerical value may change from line to line.

\section{Finite volume approximations}

Let $C_{K}^{\infty}(\mathbb{R})$ be the space of infinitely differentiable functions on $\mathbb{R}$ with compact support and introduce $\mathcal{L}=\left\{\Lambda \in C_{K}^{\infty}(\mathbb{R}): 0 \leq \Lambda \leq 1\right\}$. For $\Lambda \in \mathcal{L}$, we introduce the following finite volume approximations of the problem (1.5)

$$
m_{\Lambda}=\Lambda R-G\left[\Lambda U^{\prime}\left(m_{\Lambda}\right)\right]-\lambda \tilde{G}\left[\Lambda m_{\Lambda}\right] .
$$

In this section we establish a global existence result for (2.1) together with some bounds uniform for $\Lambda \in \mathcal{L}$. To this end we need to introduce some more notation.

For $\alpha>0$, let $\varrho(x):=e^{-\alpha|x|}$. We shall denote by $\varrho$ also the measure $\varrho(x) d x$ on $\mathbb{R}$. We introduce the following finite measures on $\mathbb{R}_{+} \times \mathbb{R}$, omitting the dependence on $\alpha>0$ from the notation

$$
\begin{aligned}
\mu(d t, d x) & :=e^{-\alpha^{2} t / 2} \varrho(x) d x d t \\
\mu_{T}(d t, d x) & :=\chi_{[0, T]}(t) \mu(d t, d x) \\
\mu_{\Lambda}(d t, d x) & :=\Lambda(x) \mu(d t, d x) \\
\mu_{T, \Lambda}(d t, d x) & :=\chi_{[0, T]}(t) \Lambda(x) \mu(d t, d x)
\end{aligned}
$$

where $\chi_{[0, T]}$ denotes the characteristic function of $[0, T]$. For $\nu$ a measure and $f$ a function use the notation $\nu(f)=\int d \nu f$.

For $p \in(1, \infty)$, we introduce the Sobolev space $H_{1}^{p}(\varrho)$ obtained by completing $C_{K}^{\infty}(\mathbb{R})$ with respect to the norm

$$
\|\varphi\|_{H_{1}^{p}(\varrho)}^{p}:=\|\nabla \varphi\|_{L^{p}(\varrho)}^{p}+\|\varphi\|_{L^{p}(\varrho)}^{p}
$$

where $\nabla$ denotes the derivative with respect to $x$. Since $H_{1}^{p}(\varrho) \subset L^{p}(\varrho)$, for $p, q \in$ $(1, \infty)$ such that $p^{-1}+q^{-1}=1$ we introduce the dual space $H_{-1}^{q}(\varrho)$ by completing 
$L^{q}(\varrho)$ with respect to the norm

$$
\|\ell\|_{H_{-1}^{q}(\varrho)}:=\sup _{\varphi:\|\varphi\|_{H_{1}^{p}(\varrho)} \leq 1} \varrho(\ell \varphi) .
$$

For $f=f(t, x)$ we shall use the notation

$$
\|f\|_{H_{-1}^{p}\left(\mu_{T}\right)}^{p}:=\int_{0}^{T} d t e^{-\alpha^{2} t / 2}\|f(t)\|_{H_{-1}^{p}(\varrho)}^{p}
$$

omitting to write $T$ on the l.h.s. if $T=\infty$.

From Lemma 1.1 it follows that for each $\alpha>0$ and $p \in[1, \infty)$ we have $Z \in L^{p}(\mu)$. Furthermore for each $T>0$ and $\Lambda \in \mathcal{L}$ we have

$$
\sup _{t \in[0, T]} \sup _{x \in \mathbb{R}}|\Lambda(x) Z(t, x)|<\infty
$$

Moreover, by the assumptions on the initial data, the same holds for

$$
R(t)=p_{t} m(0)+\lambda t p_{t} q(0)+Z(t) .
$$

We next state a local existence result for the problem (2.1). Since $\tilde{G}$ has a kernel which can be estimated as the one of $G$, the proof of the next result, which is based on Picard iterations, is the same as [3, Prop. 1] and we omit it.

Lemma 2.1 For each $\Lambda \in \mathcal{L}$ and each realization of $Z$ in $C_{\alpha}\left(\mathbb{R}_{+} \times \mathbb{R}\right)$ there is a time $T_{0}>0$ such that there exists a unique $\mathcal{F}_{t}$-adapted continuous and bounded solution of (2.1) on $\left[0, T_{0}\right] \times \mathbb{R}$.

To show the existence of a global solution, it is enough to prove that if $m_{\Lambda}$ is a continuous solution of $(2.1)$ on $\left[0, T^{*}\right) \times \mathbb{R}$ then

$$
\sup _{t \in\left[0, T^{*}\right)} \sup _{x \in \mathbb{R}}\left|m_{\Lambda}(t, x)\right|<\infty .
$$

The key ingredient for proving (2.2) is the following a priori bound on the $L^{p}\left(\mu_{T^{*}, \Lambda}\right)$ norm of solutions.

Proposition 2.2 Let $m_{\Lambda}$ be a continuous solution of (2.1) on $\left[0, T^{*}\right) \times \mathbb{R}$. For each $\alpha>0$ and $p \in[1, \infty)$ there exists a constant $C=C\left(\alpha, p,\|R\|_{L^{p}(\mu)}\right)<\infty$, independent of $T^{*}>0$ and of $\Lambda \in \mathcal{L}$, such that

$$
\left\|m_{\Lambda}\right\|_{L^{p}\left(\mu_{T^{*}, \Lambda}\right)} \leq C
$$

The proof of the proposition is split in several Lemmata, the first one, which is proven integrating by parts, is [3, Lemma 7]. 
Lemma 2.3 If both $f(t, x)$ and $\left(\partial_{t}-\frac{1}{2} \Delta\right) f$ are continuous on $(0, T) \times \mathbb{R}, f(0)=0$ and $|f|^{2 n+2}$ and $|\nabla f|^{2 n+2}$ are in $L^{1}\left(\mu_{T}\right)$, then, for any $n=0,1, \ldots$,

$$
\mu_{T}\left(f^{2 n+1}\left(\partial_{t}-\frac{1}{2} \Delta\right) f\right) \geq(2 n+1) \mu_{T}\left(f^{2 n}|\nabla f|^{2}\right)
$$

Let $m_{\Lambda}$ be a continuous solution of (2.1). We define

$$
\begin{aligned}
& u_{\Lambda}:=m_{\Lambda}-\Lambda R \\
& q_{\Lambda}:=\left(\partial_{t}-\frac{1}{2} \Delta\right) \tilde{G}\left[\Lambda m_{\Lambda}\right]
\end{aligned}
$$

From $(2.1)$, if $m_{\Lambda}$ is continuous and bounded on $[0, T] \times \mathbb{R}$, then $u_{\Lambda} \in C^{\infty}$ $((0, T] \times \mathbb{R})$ by the regularizing properties of $G$ and $\tilde{G}$. Moreover, for $t \in[0, T]$, $u_{\Lambda}(t, x)$ (together with its derivative) is exponentially decaying as $x \rightarrow \infty$.

Lemma 2.4 Let $m_{\Lambda}$ be a continuous solution of (2.1) on $\left[0, T^{*}\right) \times \mathbb{R}$; then for each $n=0,1, \ldots$ and $\beta>0$ there exists a constant $C=C(n, \beta)$, independent of $T^{*}>0$ and $\Lambda \in \mathcal{L}$, such that

$$
\mu_{T^{*}, \Lambda}\left(e^{-(2 n+2) \beta t} u_{\Lambda}^{2 n+1} U^{\prime}\left(m_{\Lambda}\right)\right) \leq C\left\|e^{-\beta t} q_{\Lambda}\right\|_{H_{-1}^{2 n+2}\left(\mu_{T^{*}}\right)}^{2 n+2}
$$

Proof. We apply Lemma 2.3 with $f=e^{-\beta t} u_{\Lambda}$ and $T<T^{*}$. From (2.1) we get

$$
\left(\partial_{t}-\frac{1}{2} \Delta\right) e^{-\beta t} u_{\Lambda}=-\beta e^{-\beta t} u_{\Lambda}-e^{-\beta t}\left[\Lambda U^{\prime}\left(m_{\Lambda}\right)+\lambda q_{\Lambda}\right]
$$

hence, by (2.4)

$$
\begin{gathered}
\mu_{T}\left(e^{-(2 n+2) \beta t}\left[(2 n+1) u_{\Lambda}^{2 n}\left(\nabla u_{\Lambda}\right)^{2}+\beta u_{\Lambda}^{2 n+2}+u_{\Lambda}^{2 n+1} \Lambda U^{\prime}\left(m_{\Lambda}\right)\right]\right) \\
\leq-\lambda \mu_{T}\left(e^{-(2 n+2) \beta t} u_{\Lambda}^{2 n+1} q_{\Lambda}\right) .
\end{gathered}
$$

Let $p, q \in(1, \infty)$ such that $p^{-1}+q^{-1}=1$; then for each $\gamma>0$ there exists a constant $C=C(\gamma, p)$ such that for any $a, b \in \mathbb{R}$

$$
|a b| \leq \gamma|a|^{p}+C|b|^{q}
$$

therefore, by the duality between $H_{1}^{p}(\varrho)$ and $H_{-1}^{q}(\varrho)$, we have

$$
|\varrho(f g)| \leq \gamma\|f\|_{H_{1}^{p}(\varrho)}^{p}+C\|g\|_{H_{-1}^{q}(\varrho)}^{q} .
$$


By applying (2.9) we get

$$
\begin{gathered}
\left|\mu_{T}\left(e^{-(2 n+2) \beta t} u_{\Lambda}^{2 n+1} q_{\Lambda}\right)\right| \leq \int_{0}^{T} d t e^{-\alpha^{2} t / 2-(2 n+2) \beta t}\left|\varrho\left(u_{\Lambda}^{2 n+1}(t) q_{\Lambda}(t)\right)\right| \\
\leq \int_{0}^{T} d t e^{-\alpha^{2} t / 2-(2 n+2) \beta t}\left[\gamma(2 n+1)^{\frac{2 n+2}{2 n+1}} \varrho\left(u_{\Lambda}(t)^{2 n \frac{2 n+2}{2 n+1}}\left(\nabla u_{\Lambda}(t)\right)^{\frac{2 n+2}{2 n+1}}\right)\right. \\
\left.+\gamma \varrho\left(u_{\Lambda}^{2 n+2}(t)\right)+C\left\|q_{\Lambda}(t)\right\|_{H_{-1}^{2 n+2}(\varrho)}^{2 n+2}\right] \\
\leq \int_{0}^{T} d t e^{-\alpha^{2} t / 2-(2 n+2) \beta t}\left[\gamma(2 n+1)^{\frac{2 n+2}{2 n+1}} c_{1}(n) \varrho\left(u_{\Lambda}(t)^{2 n}\left(\nabla u_{\Lambda}(t)\right)^{2}+u_{\Lambda}(t)^{2 n+2}\right)\right. \\
\left.+\gamma \varrho\left(u_{\Lambda}^{2 n+2}(t)\right)+C\left\|q_{\Lambda}(t)\right\|_{H_{-1}^{2 n+2}(\varrho)}^{2 n+2}\right] \\
=\gamma(2 n+1)^{\frac{2 n+2}{2 n+1}} c_{1}(n) \mu_{T}\left(e^{-(2 n+2) \beta t} u_{\Lambda}^{2 n}\left(\nabla u_{\Lambda}\right)^{2}\right) \\
+\gamma\left[(2 n+1)^{\frac{2 n+2}{2 n+1}} c_{1}(n)+1\right] \mu_{T}\left(e^{-(2 n+2) \beta t} u_{\Lambda}^{2 n+2}\right)+C\left\|e^{-(2 n+2) \beta t} q_{\Lambda}\right\|_{H_{-1}^{2 n+2}\left(\mu_{T}\right)}^{2 n+2}
\end{gathered}
$$

where we used, in the third step, Hölder inequality in the form (as follows from $(2.8))$

$$
\varrho(f g) \leq c_{1}(n)\left[\varrho\left(|f|^{\frac{2 n+1}{n+1}}\right)+\varrho\left(|g|^{\frac{2 n+1}{n}}\right)\right]
$$

with $f=\left[u_{\Lambda}(t)^{n} \nabla u_{\Lambda}(t)\right]^{\frac{2 n+2}{2 n+1}}$ and $g=u_{\Lambda}(t)^{n \frac{2 n+2}{2 n+1}}$.

Choosing $\gamma=\gamma(n, \lambda, \beta)$ small enough and taking $T \uparrow T^{*}$ the lemma now follows from (2.7) and (2.10).

Lemma 2.5 For each $p \in(1, \infty)$ there exists a constant $C=C(p)$ such that, for any $\alpha, \beta, T>0$ and $F \in L^{p}\left(\mu_{T}\right)$,

$$
\left\|e^{-\beta t}\left(\partial_{t}-\frac{1}{2} \Delta\right) \tilde{G}[F]\right\|_{H_{-1}^{p}\left(\mu_{T}\right)} \leq C\left(1+\frac{1}{\alpha}\right)\left\|e^{-\beta t} F\right\|_{L^{p}\left(\mu_{T}\right)} .
$$

Proof. We can write

$$
\tilde{G}[F](t)=\int_{0}^{t} d s p_{t-s} \int_{0}^{s} d s^{\prime} \frac{1}{2} \Delta p_{s-s^{\prime}} F\left(s^{\prime}\right)
$$

so that (in distribution sense)

$$
\left(\partial_{t}-\frac{1}{2} \Delta\right) \tilde{G}[F](t)=\int_{0}^{t} d s \frac{1}{2} \Delta p_{t-s} F(s) .
$$

We thus get

$$
\left\|\left(\partial_{t}-\frac{1}{2} \Delta\right) \tilde{G}[F](t)\right\|_{H_{-1}^{p}(\varrho)} \leq \frac{1}{2} \int_{0}^{t} d s\left\|\Delta p_{t-s} F(s)\right\|_{H_{-1}^{p}(\varrho)} .
$$


For $q^{-1}+p^{-1}=1$ and $\varphi \in C_{K}^{\infty}(\mathbb{R})$ we now have

$$
\begin{aligned}
\left(\varphi, \Delta p_{t-s} F(s)\right)_{\varrho} & =\left(\varrho \varphi, \Delta p_{t-s} F(s)\right)_{L^{2}(d x)} \\
& =-\left(\varrho\left[\nabla \varphi+\frac{\varphi \nabla \varrho}{\varrho}\right], \nabla p_{t-s} F(s)\right)_{L^{2}(d x)} \\
\leq & {\left[\|\nabla \varphi\|_{L^{q}(\varrho)}+\|\varphi\|_{L^{q}(\varrho)} \sup _{x \in \mathbb{R}}|\nabla \log \varrho(x)|\right]\left\|\nabla p_{t-s} F(s)\right\|_{L^{p}(\varrho)} }
\end{aligned}
$$

since $\sup _{x \in \mathbb{R}}|\nabla \log \varrho(x)|=\alpha$ we conclude

$$
\left\|\Delta p_{t-s} F(s)\right\|_{H_{-1}^{p}(\varrho)} \leq(1+\alpha)\left\|\nabla p_{t-s} F(s)\right\|_{L^{p}(\varrho)} .
$$

As $\exp \{-\alpha|x|\} \leq \exp \{-\alpha|y|\} \exp \{\alpha|x-y|\}$,

$$
\left|\nabla p_{t-s} F(s)(x)\right| e^{-\alpha|x| / p} \leq \int d y e^{-\alpha|y| / p}|F(s, y)|\left|\nabla p_{t-s}(x-y)\right| e^{\alpha|x-y| / p},
$$

by applying Young's inequality for convolutions we then obtain

$$
\left\|\nabla p_{t-s} F(s)\right\|_{L^{p}(\varrho)} \leq\|F(s)\|_{L^{p}(\varrho)} \int d x\left|\nabla p_{t-s}(x)\right| e^{\alpha|x| / p} .
$$

We define

$$
\psi_{\alpha, p}(t):=\int d x\left|\nabla p_{t}(x)\right| e^{\alpha|x| / p} \leq \frac{2}{\sqrt{2 \pi t}}+\frac{2 \alpha}{p} \exp \left\{\frac{\alpha^{2} t}{2 p^{2}}\right\} .
$$

Then, from (2.14), (2.16) and (2.18),

$$
\begin{gathered}
\chi_{[0, T]}(t) e^{-\frac{\alpha^{2} t}{2 p}-\beta t}\left\|\left(\partial_{t}-\frac{1}{2} \Delta\right) \tilde{G}[F](t)\right\|_{H_{-1}^{p}(\varrho)} \\
\leq \frac{1+\alpha}{2} \int d s \chi_{[0, T]}(s) e^{-\frac{\alpha^{2} s}{2 p}-\beta s}\|F(s)\|_{L^{p}(\varrho)} \chi_{[0, T]}(t-s) \\
\times e^{-\frac{\alpha^{2}(t-s)}{2 p}-\beta(t-s)} \psi_{\alpha, p}(t-s) .
\end{gathered}
$$

Again by Young's inequality for convolutions we get

$$
\begin{aligned}
\| e^{-\beta t}\left(\partial_{t}-\right. & \left.\frac{1}{2} \Delta\right) \tilde{G}[F] \|_{H_{-1}^{p}\left(\mu_{T}\right)}= \\
& \left\{\int_{0}^{T} d t e^{-\frac{\alpha^{2} t}{2}-\beta p t}\left\|\left(\partial_{t}-\frac{1}{2} \Delta\right) \tilde{G}[F](t)\right\|_{H_{-1}^{p}(\varrho)}^{p}\right\}^{1 / p} \\
\leq & \frac{1+\alpha}{2}\left\{\int_{0}^{T} d t e^{-\frac{\alpha^{2} t}{2 p}} \psi_{\alpha, p}(t)\right\}\left\{\int_{0}^{T} d t e^{-\frac{\alpha^{2} t}{2}-\beta p t}\|F(t)\|_{L^{p}(\varrho)}^{p}\right\}^{1 / p} .
\end{aligned}
$$


By using the estimate in (2.19) and recalling $p>1$, it is easy to show there is a constant $C=C(p)>0$ so that

$$
\frac{1+\alpha}{2} \int_{0}^{T} d t e^{-\frac{\alpha^{2} t}{2 p}} \psi_{\alpha, p}(t) \leq C\left(1+\frac{1}{\alpha}\right) .
$$

The lemma is proved.

Proof of Proposition 2.2. Recalling (2.5) and that $U^{\prime}(m)=m^{3}-(1-\lambda) m$, by expanding the 1.h.s. of (2.6), using Lemma 2.5, and $\Lambda^{2} \leq \Lambda$, we get there exists a constant $C=C(\alpha, n, \beta)$ such that

$$
\begin{aligned}
\mu_{T^{*}, \Lambda} & \left(e^{-(2 n+2) \beta t} u_{\Lambda}^{2 n+4}\right) \\
\leq C \mu_{T^{*}, \Lambda}\left(e^{-(2 n+2) \beta t}\{\mid\right. & \left.u_{\Lambda}\right|^{2 n+1}\left[u_{\Lambda}^{2}|\Lambda R|+\left|u_{\Lambda}\right|(\Lambda R)^{2}+|\Lambda R|^{3}\right] \\
& \left.\left.+u_{\Lambda}^{2 n+2}+\left|u_{\Lambda}\right|^{2 n+1}|\Lambda R|+|\Lambda R|^{2 n+2}\right\}\right) .
\end{aligned}
$$

Let

$$
M:=\left(\mu_{T^{*}, \Lambda}\left(e^{-(2 n+2) \beta t} u_{\Lambda}^{2 n+4}\right)\right)^{1 /(2 n+4)}
$$

by using repeatedly Hölder inequality in (2.20) we get

$$
\begin{aligned}
M^{2 n+4} \leq C\{ & M^{2 n+3}\|\Lambda R\|_{L^{2 n+4}(\mu)}+M^{2 n+2}\left[1+\|\Lambda R\|_{L^{2 n+4}(\mu)}^{2}\right] \\
& \left.+M^{2 n+1}\|\Lambda R\|_{L^{2 n+4}(\mu)}\left[1+\|\Lambda R\|_{L^{2 n+4}(\mu)}^{2}\right]+\|\Lambda R\|_{L^{2 n+4}(\mu)}^{2 n+2}\right\}
\end{aligned}
$$

we then conclude that $M$ is bounded by some constant $C=C(\alpha, n, \beta$, $\left.\|\Lambda R\|_{L^{2 n+4}(\mu)}\right)$.

Recalling $m_{\Lambda}=u_{\Lambda}+\Lambda R$, by using triangular and Cauchy-Schwartz inequalities, we have

$$
\left\|m_{\Lambda}\right\|_{L^{p}\left(\mu_{\Lambda, T^{*}}\right)} \leq\|\Lambda R\|_{L^{p}\left(\mu_{\Lambda, T^{*}}\right)}+\left\|e^{\beta t}\right\|_{L^{2 p}\left(\mu_{\Lambda, T^{*}}\right)}\left\|e^{-\beta t} u_{\Lambda}\right\|_{L^{2 p}\left(\mu_{\Lambda, T^{*}}\right)} .
$$

Since, for each $p \in[1, \infty),\|\Lambda R\|_{L^{p}\left(\mu_{\Lambda, T^{*}}\right)} \leq\|R\|_{L^{p}(\mu)}<\infty$, by choosing $\beta=$ $\beta(\alpha, p)$ small enough and $n=n(p)$ large enough, the proposition follows.

Proof of (2.2). Let $Y^{\star}:=\sup \{|x|: \Lambda(x)>0\}$; writing explicitly the kernels in the integral equation (2.1), and using Young's inequality for convolutions, it follows

$$
\begin{aligned}
& \sup _{t \in\left[0 . T^{*}\right)} \sup _{x \in \mathbb{R}}\left|m_{\Lambda}(t, x)\right| \leq \sup _{t \in\left[0, T^{*}\right)} \sup _{x \in \mathbb{R}}|\Lambda(x) R(t, x)| \\
& +e^{\frac{\alpha^{2}}{4} T^{*}+\frac{\alpha}{2} Y^{*}} \times\left\{\left[\int_{0}^{T^{*}} d t \int d z p_{t}(z)^{2}\right]^{\frac{1}{2}}\left[\int \mu_{T^{*}, \Lambda}(d t, d x) U^{\prime}\left(m_{\Lambda}(t, y)\right)^{2}\right]^{\frac{1}{2}}\right. \\
& \left.+\lambda\left[\int_{0}^{T^{*}} d t \int d z p_{t}(z)^{2}\left[\frac{z^{2}}{2 t}+\frac{1}{2}\right]^{2}\right]^{\frac{1}{2}}\left[\int \mu_{T^{*}, \Lambda}(d t, d x) m_{\Lambda}(t, y)^{2}\right]^{\frac{1}{2}}\right\}
\end{aligned}
$$


where we used also that $\Lambda^{2} \leq \Lambda$. By using that, as follows from Proposition 2.2, $U^{\prime}\left(m_{\Lambda}\right)$ is in $L^{2}\left(\mu_{T^{*}, \Lambda}\right)$, and the properties of the heat kernel, it is easy to see that the r.h.s. of (2.21) is bounded.

The following lemma states an a priori uniform bound for the solutions of (2.1), which by (2.2) are defined for any $t \geq 0$.

Lemma 2.6 Let $m_{\Lambda}$ be a continuous solution of (2.1) on $\mathbb{R}_{+} \times \mathbb{R}$. Then for each $\alpha>0, p \in[1, \infty)$ there exists a constant $C=C\left(\alpha, p,\|R\|_{L^{p}(\mu)}\right)<\infty$ independent of $\Lambda \in \mathcal{L}$ such that $\left\|m_{\Lambda}\right\|_{L^{p}(\mu)} \leq C$.

Proof. Since the continuous solution of equation (2.1) exist globally in time, the inequality (2.3) may be extended for $T^{*} \uparrow \infty$; we get

$$
\left\|\Lambda m_{\Lambda}\right\|_{L^{p}(\mu)} \leq\left\|m_{\Lambda}\right\|_{L^{p}\left(\mu_{\Lambda}\right)} \leq C<\infty .
$$

Note that for $p>1$ both $G$ and $\tilde{G}$ are bounded operators in $L^{p}(\mu)$ (see [3, Lemma 9] for $G$ and the same proof also works for $\tilde{G}$ ). From the integral equation (2.1), the bound (2.22), and $R \in L^{p}(\mu)$ the lemma follows.

\section{Infinite volume equation}

In this section we conclude the proof of Theorem 1.3 by removing the truncation $\Lambda$ in (2.1); following [3], we prove first that, provided $\beta$ is chosen large enough, $\exp (-\beta t) \Lambda m_{\Lambda}$ converges in $L^{p}(\mu)$.

Lemma 3.1 Let $m_{\Lambda}$ be a solution of (2.1). For each $p \in[1, \infty)$ there exists a positive constant $k_{p}<\infty$ such that, for any $\alpha>0$ and $\beta \geq k_{p}\left(1+\alpha^{-p}\right)$, $\left\{\exp (-\beta t) \Lambda m_{\Lambda}, \Lambda \in \mathcal{L}\right\}$ is Cauchy in $L^{p}(\mu)$ as $\Lambda \uparrow 1$.

Proof. Recalling (2.5), if we consider $f=e^{-\beta t}\left[u_{\Lambda}-u_{\bar{\Lambda}}\right]$, from equation (2.1),we have

$$
\left(\partial_{t}-\frac{1}{2} \Delta\right) f=-\beta f-e^{-\beta t}\left[\Lambda U^{\prime}\left(m_{\Lambda}\right)-\bar{\Lambda} U^{\prime}\left(m_{\bar{\Lambda}}\right)\right]-\lambda e^{-\beta t}\left[q_{\Lambda}-q_{\bar{\Lambda}}\right] .
$$

By the same computations as in Lemma 2.4 with $f$ as above, (see equations (2.7) and (2.10)), but choosing $\gamma=\gamma(n, \lambda)$ independent of $\beta$ and taking $T \uparrow \infty$, there exists a constant $c_{1}=c_{1}(n)$ independent of $\alpha, \beta$ such that

$$
\begin{aligned}
& \mu\left(e^{-(2 n+2) \beta t}\left[u_{\Lambda}-u_{\bar{\Lambda}}\right]^{2 n+1}\left[\Lambda U^{\prime}\left(m_{\Lambda}\right)-\bar{\Lambda} U^{\prime}\left(m_{\bar{\Lambda}}\right)\right]\right) \\
& +(\beta-1) \mu\left(e^{-(2 n+2) \beta t}\left[u_{\Lambda}-u_{\bar{\Lambda}}\right]^{2 n+2}\right) \leq c_{1}\left\|e^{-\beta t}\left(q_{\Lambda}-q_{\bar{\Lambda}}\right)\right\|_{H_{-1}^{2 n+2}(\mu)}^{2 n+2} .
\end{aligned}
$$


By using Lemma 2.5 there is a constant $c_{2}=c_{2}(n)$ such that

$$
\begin{aligned}
\| e^{-\beta t}\left(q_{\Lambda}-q_{\bar{\Lambda}}\right) & \|_{H_{-1}^{2 n+2}(\mu)}^{2 n+2} \\
& \leq c_{2}\left(1+\alpha^{-(2 n+2)}\right) \mu\left(e^{-(2 n+2) \beta t}\left[\Lambda m_{\Lambda}-\bar{\Lambda} m_{\bar{\Lambda}}\right]^{2 n+2}\right) .
\end{aligned}
$$

On the other hand, since for any $a, b \in \mathbb{R}$ we have $\left(a^{3}-b^{3}\right)(a-b) \geq(a-b)^{4} / 4$, there exists a constant $c_{3}=c_{3}(\lambda)$ such that

$$
\begin{aligned}
& \left(u_{\Lambda}-u_{\bar{\Lambda}}\right)\left[\Lambda U^{\prime}\left(m_{\Lambda}\right)-\bar{\Lambda} U^{\prime}\left(m_{\bar{\Lambda}}\right)\right] \\
& =\left(m_{\Lambda}-m_{\bar{\Lambda}}-(\Lambda-\bar{\Lambda}) R\right)\left(\Lambda m_{\Lambda}^{3}-\bar{\Lambda} m_{\bar{\Lambda}}^{3}\right)-(1-\lambda)\left(u_{\Lambda}-u_{\bar{\Lambda}}\right)\left(\Lambda m_{\Lambda}-\bar{\Lambda} m_{\bar{\Lambda}}\right) \\
& \quad \geq \frac{1}{4}\left(\Lambda m_{\Lambda}-\bar{\Lambda} m_{\bar{\Lambda}}\right)^{4}-c_{3}\left(u_{\Lambda}-u_{\bar{\Lambda}}\right)^{2} \\
& \quad-c_{3}(|1-\Lambda|+|1-\bar{\Lambda}|)\left[m_{\Lambda}^{2}\left(1+m_{\Lambda}^{2}\right)+m_{\bar{\Lambda}}^{2}\left(1+m_{\bar{\Lambda}}^{2}\right)+R^{2}\left(1+R^{2}\right)\right]
\end{aligned}
$$

By plugging the bounds (3.2) and (3.3) into (3.1) and using Hölder inequality, we find there exists a $c_{4}=c_{4}(n, \alpha, \lambda)$ such that

$$
\begin{aligned}
& \frac{1}{4} \mu\left(e^{-(2 n+2) \beta t}\left(\Lambda m_{\Lambda}-\bar{\Lambda} m_{\bar{\Lambda}}\right)^{2 n+4}\right) \\
& +\left(\beta-1-c_{3}-c_{1} c_{2}\left(1+\alpha^{-(2 n+2)}\right)\right) \mu\left(e^{-(2 n+2) \beta t}\left(u_{\Lambda}-u_{\bar{\Lambda}}\right)^{2 n+2}\right) \\
& \leq c_{4}\left(\|1-\Lambda\|_{L^{2}(\mu)}+\|1-\bar{\Lambda}\|_{L^{2}(\mu)}\right) \\
& \quad \times\left\{1+\left\|m_{\Lambda}\right\|_{L^{4 n+8}(\mu)}^{2 n+4}+\left\|m_{\bar{\Lambda}}\right\|_{L^{4 n+8}(\mu)}^{2 n+4}+\|R\|_{L^{4 n+8}(\mu)}^{2 n+4}\right\} .
\end{aligned}
$$

Given $p \geq 2$, let $n=n(p)=[p / 2]-1$ and $k_{p}$ such that $1+c_{3}+c_{1} c_{2}\left(1+\alpha^{-(2 n+2)}\right)$ $\leq k_{p}\left(1+\alpha^{-p}\right)$ for any $\alpha>0$. The lemma now follows from Lemma 2.6 and $R \in L^{p}(\mu)$ for any $p \in[1, \infty)$.

The proof of Theorem 1.3 can now be completed as in [3], we shall just sketch the argument.

Proof of Theorem 1.3. To prove existence of a continuous solution of (1.5), we first note that, for each $p \in[1, \infty)$ and $\alpha$ so large that $\alpha^{2}>k_{p}\left(1+\alpha^{-p}\right), m_{\Lambda}$ is Cauchy in $L^{p}(\mu)$. This follows from Lemmata 2.6, 3.1, and Hölder inequality; moreover the limit $m$ satisfies equation (1.5). Since $G$ and $\tilde{G}$ map $L^{2}(\mu)$ into $C_{\alpha}\left(\mathbb{R}_{+} \times \mathbb{R}\right)$, see $\left[3\right.$, Lemma 12], we also have $m \in C_{\alpha}\left(\mathbb{R}_{+} \times \mathbb{R}\right)$.

In order to show $m \in C_{\alpha}\left(\mathbb{R}_{+} \times \mathbb{R}\right)$ for any $\alpha>0$, we note that, by Lemma 2.6, $m_{\Lambda}$ is uniformly bounded in $L^{p}(\mu)$; we can thus find a weakly convergent subsequence $m_{\Lambda_{k}} \rightarrow m^{\prime}$. On the other hand, by Lemma 3.1, $e^{-\beta t} \Lambda_{k} m_{\Lambda_{k}}$ converges strongly in $L^{p}(\mu)$ for $\beta \geq k_{p}\left(1+\alpha^{-p}\right)$, hence $m=m^{\prime} \mu$-a.s. Since $m^{\prime} \in L^{p}(\mu)$ for any $\alpha>0$ and $p \in[1, \infty)$, by the same argument as above, we get $m^{\prime} \in C_{\alpha}\left(\mathbb{R}_{+} \times \mathbb{R}\right)$ and $m=m^{\prime}$.

To prove uniqueness, let $m_{1}$ and $m_{2}$ be two continuous solutions of (1.5). By applying Lemma 2.3 to the function $f=e^{-\beta t}\left[m_{1}-m_{2}\right]$ and repeating the same computations as in Lemma 3.1 it is easy to show $m_{1}=m_{2}$. 
Acknowledgments. L. Bertini acknowledges the very kind hospitality of Departamento de Matemáticas, Instituto Venezolano de Investigaciones Científicas.

\section{References}

[1] L. Bertini, S. Brassesco, P. Buttà and E. Presutti, Front fluctuations in one dimensional stochastic phase field equations, Ann. Henri Poincaré 3, 29-86 (2002).

[2] G. Caginalp and X. Chen, Convergence of the phase field model to its sharp interface limits, European J. Appl. Math. 9, 417-445 (1998).

[3] C. R. Doering, Nonlinear parabolic stochastic differential equations with additive colored noise on $\mathbb{R}^{d} \times \mathbb{R}_{+}$: a regulated stochastic quantization, Comm. Math. Phys. 109, 537-561 (1987).

[4] W. G. Faris and G. Jona-Lasinio, Large fluctuations for a nonlinear heat equation with noise, J. Phys. A 15, 3025-3055 (1982).

[5] P. C. Hohenberg and B. I. Halperin, Theory of dynamic critical phenomena, Rev. Mod. Phys. 49, 435-479 (1977).

[6] H. M. Soner, Convergence of the phase-field equations to the Mullins-Sekerka problem with kinetic undercooling, Arch. Rational Mech. Anal. 131, 139-197 (1995).

Lorenzo Bertini

Dipartimento di Matematica

Università di Roma La Sapienza

Piazzale Aldo Moro 2

00185 Roma

Italy

email: lorenzo@carpenter.mat.uniroma1.it

Paolo Buttà

Dipartimento di Matematica

Università di Roma La Sapienza

Piazzale Aldo Moro 2

00185 Roma

Italy

email: butta@mat.uniroma1.it
Stella Brassesco

Departamento de Matemáticas

Instituto Venezolano de

Investigaciones Científicas

Apartado Postal 21827

Caracas 1020-A

Venezuela

email: sbrasses@ivic.ivic.ve

Errico Presutti

Dipartimento di Matematica

Università di Roma Tor Vergata

Via della Ricerca Scientifica

00133 Roma

Italy

email: presutti@mat.uniroma2.it

Communicated by Jean-Pierre Eckmann submitted 30/01/01, accepted 13/06/01 